\title{
Periorbital edema from apixaban treatment
}

\author{
Amier Ahmad, $M D^{7}$ and Starr Steinhillber, $M D, M P H^{1,2}$ \\ 'Department of Medicine, Tinsley Harrison Internal Medicine Residency Program, The University of Alabama at Birmingham, Birmingham, AL, USA; \\ ${ }^{2}$ Division of General Internal Medicine, Department of Medicine, The University of Alabama at Birmingham, Birmingham, AL, USA.
}

KEY WORDS: apixaban; periorbital edema; side effect.

J Gen Intern Med 33(2):232

DOI: $10.1007 / \mathrm{s} 11606-017-4180-1$

(C) Society of General Internal Medicine 2017

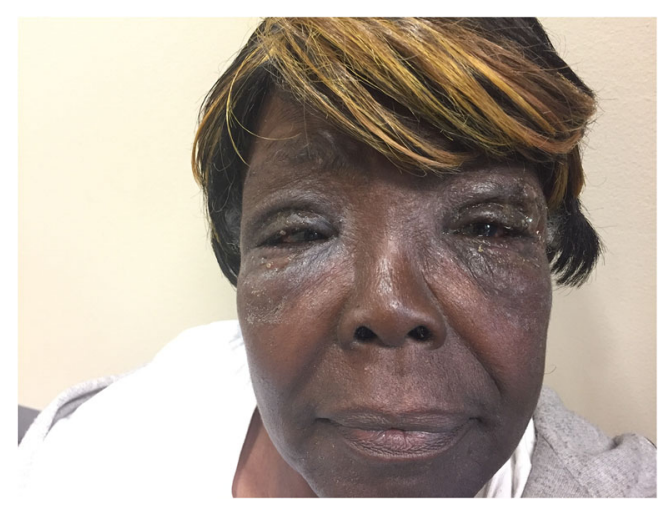

Figure 1 Bilateral periorbital edema 2 weeks after initiation of apixaban

\section{CASE PRESENTATION}

A 70-year-old female with nonvalvular atrial fibrillation presented with 2 weeks of progressive bilateral periorbital swelling and pruritus limited to her eyes. Her symptoms started 2 days after her oral anticoagulation was changed from warfarin to apixaban. She denied rash, shortness of breath, and tongue or lip swelling. She had made no other medication changes and had used no new facial products within that time period. Examination revealed bilateral eyelid edema with chemosis, tearing, and conjunctival hyperemia (Fig. 1). Her apixaban was discontinued and she was referred to ophthalmology. There were no visual changes on formal ophthalmologic examination, and the patient was instructed to apply triamcinolone cream locally for symptomatic relief. Within

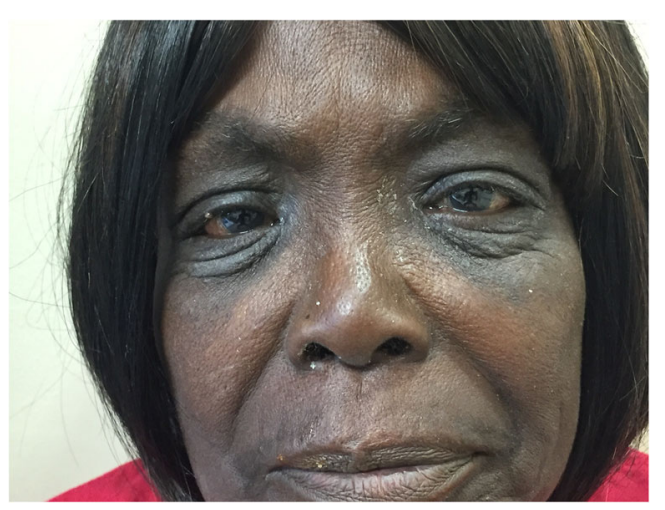

Figure 2 Resolution of periorbital edema after apixaban discontinuation

1 week, her symptoms had resolved (Fig. 2). She was subsequently started on rivaroxaban without issue.

Apixaban is a direct inhibitor of factor $\mathrm{Xa}$ for which no hypersensitivity reactions have been reported in the literature (other than product information reporting a frequency of $0.1-$ $1 \%$ ). The underlying mechanism resulting in periorbital edema remains unclear and is not established in the literature. Ours is the first report of bilateral periorbital edema likely from the use of apixaban with complete resolution of symptoms after cessation of the drug.

Financial support: None.

Corresponding Author: Amier Ahmad, MD; Department of Medicine, Tinsley Harrison Internal Medicine Residency ProgramThe University of Alabama at Birmingham, BDB 327, 1720 2nd Ave South, Birmingham, AL 35294, USA (e-mail: aahmad@uabmc.edu).

Compliance with ethical standards:

Conflict of interest: The authors declare no conflicts of interest. 